\title{
Valor simbólico de los objetos para vestir la sala comedor del hogar y su aporte a la apropiación de los espacios
}

\begin{abstract}
Resumen
La apropiación del espacio es un proceso que permite hacer lecturas de cambios en las relaciones al interior de la familia entre los individuos, los objetos y el espacio mismo. En este sentido es posible encontrar diferentes representaciones y prácticas - para vestir los espacios del hogar y en especial la sala comedor-que permiten escenarios de simbolización, proyección e interacción en el ámbito íntimo o familiar. En esta investigación se estudió cualitativamente a partir de entrevistas y observación el valor simbólico de los objetos para vestir la sala comedor en las familias de nivel socioeconómico 3 y 4 de Sabaneta, Antioquia, para comprender el aporte de estos a la apropiación de los espacios. La investigación evidencia que el consumo de los espacios modernos y especialmente de la sala comedor se ha resignificado apelando a una relación de placer, de disfrute individual, familiar y social; en donde las normas colectivas no han impedido el desarrollo de nuevas prácticas de vestir este espacio, lo que permite la proyección de identidad y materializa además el confort por medio de objetos multifuncionales y adaptables a la sala comedor. En esta dinámica de apropiación los individuos a través del uso y disposición de los objetos establecen relaciones con estos y los van cargando de significado, de vivencias y símbolos en busca del disfrute colectivo o individual, de lo estético y sensitivo y del bienestar sensorial.
\end{abstract}

\author{
Viviana Montoya Cataño \\ Magíster en Comportamiento del \\ Consumidor \\ Universidad Pontificia Bolivariana \\ Medellín, Colombia \\ ๑ orcid.org/0000-0001-9084-0705 \\ Google Scholar \\ Isabel Cristina Torres Estrada \\ Magíster en Desarrollo \\ Docente Universidad Pontificia \\ Bolivariana \\ Medellín, Colombia \\ Correo electrónico: isabel.torres@ \\ upb.edu.co \\ ๑ orcid.org/0000-0003-1940-2731 \\ Google Scholar
}

Recibido: Diciembre 15 de 2017

Aprobado: Agosto 20 de 2018

Palabras clave:

Apropiación del espacio, consumo, objetos, sala comedor, valor simbólico. 


\section{Symbolic value of the objects to dress the living-dining room at home and its contribution to the appropriation of the spaces}

\begin{abstract}
The appropriation of space is a process that allows readings of changes in relationships within the family, between individuals, objects and space itself. In this sense it is possible to find different representations and practices to dress the spaces of the home and especially the living-dining room, allowing scenarios of symbolization, projection and interaction in the intimate or family environment. In this research, the symbolic value of the objects to dress the living-dining room in homes of 3 and 4 socioeconomic level families in Sabaneta, Antioquia, were qualitatively studied from interviews and observation to understand their contribution to the appropriation of spaces. The research shows that the consumption of modern spaces and especially the living-dining room has been resignified by appealing to a relationship of pleasure, individual, family and social enjoyment, where the collective norms have not prevented the development of new practices of dressing this space, which allows the projection of identity and also materializes comfort by means of multifunctional objects adaptable to the living-dining room. In this dynamic of appropriation, through the use and disposition of objects, individuals establish relationships with them and charge them with meaning, experiences and symbols in search of collective or individual enjoyment, of the aesthetic and sensory, and of the sensory well-being.
\end{abstract}

Key words:

Appropriation of space, consumption, dining room, objects, symbolic value. 


\section{Introducción}

Los objetos no tienen significado en sí mismos sino que lo adquieren de acuerdo al uso y al valor que las personas les dan. En este sentido están inmersos en sistemas de representaciones, prácticas y relaciones de las que son parte. "Desde el estructuralismo se reconoce cómo las posesiones materiales llevan implícitos significados actuando como comunicadores de la cultura" (Lévi-Strauss, 1988, p. 38). Además de comunicar, los objetos juegan un rol fundamental en la apropiación simbólica de los espacios y en la configuración de estos. Para Chávez (2010):

\footnotetext{
el espacio arquitectónico doméstico, además de otras cosas, es la extensión de la piel; es, al igual que el vestido, el territorio expandido del ser para protección, disfraz, comunicación y relación. Tanto la arquitectura - y, sobre todo, el espacio arquitectónico doméstico-como el vestido nos comunican, nos hablan, y somos hablados por ellos, y como tales, portadores de mensajes; poseen una enorme capacidad semántica y simbólica, y son además receptores de memoria y de futuro. (p. 12)
}

Asimismo, los objetos para vestir la sala comedor poseen ese simbolismo e inciden en la apropiación de los espacios que se dan en un proceso de constante transformación al igual que las reconfiguraciones familiares. A nivel nacional y mundial se han estudiado las reconfiguraciones de las familias, los espacios domésticos, la injerencia de estos en las relaciones familiares y en los roles de los miembros que las integran; sin embargo no se ha investigado la conexión que poseen las construcciones simbólicas para vestir la sala comedor del hogar y su incidencia en el fenómeno en la apropiación contemporánea de los espacios. Igualmente "jamás podremos explicar la demanda si sólo consideramos las propiedades físicas de las mercancías. El hombre las necesita para comunicarse con otros hombres y para dar sentido a lo que sucede a su alrededor" (Douglas, 1979, p. 111). El consumo además de tener un valor simbólico, cumple una función de transferir y comunicar significados. 
Por otra parte, como refiere Sassatelli (2012),

el consumo es sin lugar a duda, una cuestión de gustos, pero los gustos no son lo único que cuenta para comprender nuestros deseos: los lugares, los tiempos y las formas de consumo son otras tantas estructuras sociales en relación con las cuales los gustos se traducen en la práctica. (p. 217)

Por ello es fundamental reconocer que las prácticas y representaciones están cargadas de un valor simbólico que incide en las configuraciones no solo de las relaciones, sino también en la apropiación de los espacios.

Por esta razón, es ineludible que las marcas hagan una lectura correcta en los terrenos simbólicos e inconscientes que componen las representaciones y prácticas en la apropiación de los espacios domésticos que a la vez se han venido transformando. Tal es el caso del municipio de Sabaneta (Antioquia) que, según el Plan de Desarrollo Municipal 2016-2019, su crecimiento en los últimos 10 años desborda los cálculos y tendencias; en catastro municipal no se tiene una cifra actualizada del total de los predios puesto que el ritmo acelerado de las construcciones no coincide con el de las incorporaciones de nuevos predios que en su mayoría son edificios de apartamentos que pueden dar cuenta de esas nuevas dinámicas de los objetos en la sala comedor, su simbolismo, la apropiación del espacio y su relación con el consumo.

Con todo lo anterior, vale la pena preguntarse: ¿cómo el valor simbólico de los objetos para vestir la sala comedor, de los hogares de estrato 3 y 4 de Sabaneta, inciden en la apropiación de los espacios?

La presente investigación articula desde la perspectiva del consumo dos conceptos: los objetos y el espacio; comprendiendo que los objetos además de su función, tiempo, uso privado, público, familiar o individual están cargados de un valor simbólico que incide en la apropiación no solo de las relaciones, sino también de los espacios. Según Bal (2009), la significación de los objetos 
"llega desde afuera, desde el marco cultural" (p. 63). Partiendo de esto, las personas se relacionan con los objetos y estos a la vez con ellos mismos: "sin relación no hay espacio, pues el espacio no existe sino abierto, suscitado, ritmado, ampliado por una correlación de los objetos y un rebasamiento de su función en esta nueva estructura" (Baudrillard, 1978, p. 17). Tal relación como ya se ha mencionado puede darse en el terreno público o privado, haciendo referencia al espacio que está demarcado por el hogar; pues el solo hecho de entrar en la morada conduce al espacio de lo propio, lo personal e íntimo.

El hogar, bajo esta mirada tradicional, tal y como se ha observado, es considerado como el espacio que forja el espacio del adentro, la privacidad y, por consiguiente, la seguridad y la estereotipia de roles. La morada es vista como espacio que genera identidad y, por esa razón, se la valora por lo que aporta al sujeto. (Cano, 2012, p. 103)

Históricamente el hogar se ha definido como el lugar en el que el sujeto lleva a cabo su existencia de manera más íntima pero también denotando una vida en común con familiares, pareja y amigos. De igual manera la existencia de los objetos no es aislada dado que su naturaleza simbólica nos lleva a considerar una óptica relacional que no solo refleja la organización de estos en la vida de los individuos, sino también el simbolismo y representaciones que se materializan en la configuración de los espacios del hogar. Desde esta perspectiva:

la sala es un territorio interesante pues es una especie de portal entre lo público que arriba para entrometerse en lo privado y, a la vez, un límite, una zona de control del intruso. Esta duplicidad en la definición de la sala misma auxilia la posibilidad de identificar de qué manera los objetos de uso disponibles en la sala incorporan significaciones y valores culturales al interior de la vida cotidiana. (Pineda, 2016, p. 72)

Y es en este espacio donde los objetos que la 'visten' poseen un simbolismo y están relacionados con las configuraciones de los espacios que se dan en un proceso de constante transformación, donde el orden simbólico de los 
objetos cambia al igual que las relaciones al interior de la familia; algunos se substituyen, otros evolucionan y permiten nuevas formas de apropiación y otros se aíslan:

antropomórficos, estos dioses lares que son los objetos se vuelven, al encarnar en el espacio los lazos afectivos y la permanencia del grupo, suavemente inmortales hasta que una generación moderna los relega o los dispersa, o a veces los reinstaura en una actualidad nostálgica de objetos viejos. (Baudrillard, 1978, p. 14)

Además de ello este proceso de transformación, como refiere Lipovetsky (2006), ha pasado de una lógica de consumo de tipo semicolectivo - basada en el equipamiento del hogar- a un equipamiento de los individuos que integran el hogar. Estas prácticas de consumo más individualizadas generan cambios en las dinámicas familiares, en el modo de organización de la vida privada y en el uso personalizado del espacio, del tiempo y de los objetos.

Así estas nuevas dinámicas familiares y de consumo se hallan inmersas en el simbolismo que, como lo refiere Varela,

es una propiedad inherente a la percepción de los espacios, donde el significado puede derivar de las características físico-estructurales, de la funcionalidad ligada a las prácticas sociales que en éstos se desarrollan o de las interacciones simbólicas entre los sujetos que ocupan dicho espacio. (Como se citó en Vidal y Pol, 2005, p. 286)

Es por todo esto que estudiar el espacio de la sala comedor ayuda a comprender las estructuras sociales y de relación de una época. Ahora bien, profundizar en la comprensión de ello - vinculando además su relación con los objetos y sus representaciones al momento de vestir la sala comedor- permitirá interpretar la conexión de este fenómeno a la luz de la apropiación de estos en relación al consumo. 


\section{Método}

Esta investigación se fundamentó en el enfoque cualitativo, el cual además de realizar una descripción de los fenómenos sociales que investiga permite construir cadenas de interpretación que ayudan a una mejor explicación de la realidad social de la que se ocupa. La perspectiva metodológica utilizada fue el interaccionismo simbólico, ya que este hace gran énfasis en la importancia del significado e interpretación como procesos humanos esenciales en los que las personas crean significados compartidos a través de su interacción y estos significados devienen su realidad.

Debido a que el objetivo del estudio fue dar cuenta del valor simbólico de los objetos para "vestir la sala comedor" de los hogares estrato 3 y 4 de Sabaneta, y su aporte a la apropiación de los espacios, los criterios de selección de los sujetos de estudio se enfocaron en estos niveles socioeconómicos; se buscó diversidad en las tipologías de familias, seleccionando: 2 hogares de familias nucleares; 2 de familias monoparentales; 2 de familias conformadas solo por hermanos. Por su parte el tipo de vivienda estuvo representada por apartamentos. La muestra fue de máxima variación y se llegó a las unidades de análisis por bola de nieve, según los criterios de selección descritos. El momento de finalización para la recolección de datos se definió bajo el criterio de saturación teórica. Con respecto a las técnicas de recolección de información se realizaron seis entrevistas a profundidad y observación. Considerando que "el sentido de la vida social se expresa particularmente a través de discursos que emergen constantemente en la vida diaria, de manera informal por comentarios, anécdotas, términos de trato y conversaciones" (Guber, 2001, p. 75). La entrevista fue la técnica principal de recolección de datos, donde las verbalizaciones se obtuvieron por medio de la observación directa y participación, abordando los temas de la investigación de una manera más profunda y tranquila mediante una conversación espontánea, menos dirigida, 
dentro de ese espacio de la sala comedor de las personas investigadas; en ese sentido fue válido que intervinieran otras personas del grupo familiar o que el entrevistado tuviera conversaciones o actividades propias de su cotidianidad. Durante el trabajo de campo se realizaron anotaciones en diarios de campo percibiendo emociones, significados e interacciones; además se hizo una descripción de los espacios con fotografías y se construyó una relación de objetos con sus significados. Las categorías previas a partir de las cuales se diseñó la guía de entrevista fueron: a cerca de la familia y sus actividades; a cerca de los espacios del hogar y a cerca de la sala comedor. Por su parte las categorías de la observación fueron: contexto familiar y vida diaria; contexto vivienda; rol de la sala comedor; objetos de la sala comedor.

\section{Resultados}

De acuerdo con la propuesta de análisis de los objetos de Medina (2005) estos se pueden estudiar a partir de su taxonomía, eje temporal, paradigma y sintagma, la primera y segunda piel del objeto, los ejes y la estructura. Con base en ello se describen los principales hallazgos identificados en los objetos de la sala comedor de las familias entrevistadas y su relación con el espacio, el consumo y la práctica. 
Tabla 1. Relación espacio/objeto/práctica/consumo.

\begin{tabular}{|c|c|c|c|}
\hline Espacio & Objeto & Práctica & Consumo \\
\hline Público & $\begin{array}{l}\text { Confortable } \\
\text { Decorativo } \\
\text { Entretiene } \\
\text { Multifuncional } \\
\text { Tecnología/lujo } \\
\text { Algunos objetos } \\
\text { tradicionales desaparecen }\end{array}$ & Socialización, interacción & $\begin{array}{l}\text { Se convierte en un espacio } \\
\text { multifuncional } \\
\text { Lo antiguo y lo heredado } \\
\text { queda relegado } \\
\text { De objetos para mascotas }\end{array}$ \\
\hline Privado & $\begin{array}{l}\text { Personalización } \\
\text { Confortable } \\
\text { Decorativo } \\
\text { Entretiene } \\
\text { Multifuncional } \\
\text { Tecnología/lujo } \\
\text { Algunos objetos } \\
\text { tradicionales desaparecen } \\
\text { Poca presencia de objetos } \\
\text { religiosos } \\
\text { Reflejan espiritualidad }\end{array}$ & $\begin{array}{l}\text { Compartir alimentos y } \\
\text { momentos }\end{array}$ & $\begin{array}{l}\text { Objetos con más estética, } \\
\text { tecnología y funcionalidad }\end{array}$ \\
\hline Individual & $\begin{array}{l}\text { Personalización } \\
\text { Confortable } \\
\text { Entretiene } \\
\text { Multifuncional } \\
\text { Tecnología/lujo }\end{array}$ & $\begin{array}{l}\text { Trabajo, estudio, } \\
\text { entretenimiento }\end{array}$ & \\
\hline
\end{tabular}

Fuente: elaboración propia por parte de las autoras.

\section{Apropiación de los espacios}

El significado del espacio procede en gran medida de las experiencias que acontecen en él: "la experiencia emocional en los lugares implica que las acciones que se desarrollan en el lugar y las concepciones que del lugar se generan están imbricadas" (Vidal y Pol, 2005, p. 288), entonces parte del significado se deriva de las interacciones simbólicas que se dan en este. En los 
hogares estudiados se evidenciaron 3 tipos de interacciones entre los sujetos que son relevantes y que también suceden en el espacio de la sala comedor que representa para ellos un lugar de encuentro donde converge toda la familia, se comparten las comidas, las conversaciones, los momentos de relajación y esparcimiento, se atienden las visitas y a los amigos; y aparte de esto es un lugar muy funcional para trabajar, estudiar, descansar, tener momentos de ocio, momentos individuales y colectivos.

La primera es la interacción familiar: el diálogo, el compartir actividades del día a día, las vivencias y conversaciones en general acontecen en este espacio. En la mañana el desayuno, discursos y rutinas antes de salir del hogar empiezan en el comedor; ya en la noche los miembros del hogar se reúnen en la sala a compartir nuevamente conversaciones y vivencias de lo que aconteció en el día, en el trabajo, en el estudio entre otros. El comedor es muy valorado por el compartir que permite en cualquiera de las comidas: "por ejemplo hoy nos sentamos los cuatro en la mesa, eso me parece genial poder compartir el almuerzo, o el desayuno, o la comida, así no se hable un tema específico, pero me parece muy chévere cuando podemos coincidir" (Hog. 6); se convierte en un momento de unión familiar. Por su parte la sala es ese espacio en la que al llegar del trabajo, de la calle, se convoca la familia a compartir; llegan algunos miembros de la familia a descansar, otros ven televisión juntos, otros conversan, otros esperan mientras está la cena; es el lugar donde se da esa interacción familiar que antecede a un momento más individual de cada miembro que acontece en su habitación. Porque definitivamente esa apropiación de la sala comedor coincide en la mayoría de los casos con una interacción colectiva: "cuando estoy sola, estoy más tiempo en mi habitación, cuando estamos las dos si estamos más en la sala comedor" (Hog. 4).

La segunda interacción evidenciada en la sala comedor es la social: las visitas, las reuniones con los amigos, los almuerzos y cenas, las tertulias, todo sucede 
en este espacio; que en los apartamentos modernos está integrado incluso con la cocina, permitiendo mayor interacción: "cuando vienen amigos, nos sentamos ahí, a tomar, a comer, hacemos una comida, y como te digo, como la sala comedor queda al lado de la cocina mientras unos comparten otros cocinan. En realidad, esta es como la zona social del apartamento" (Hog. 4). Esto sucede sobre todo los fines de semana en el que las rutinas cambian, hay mayor interacción en este espacio porque la familia está más tiempo, además llegan los invitados para compartir momentos de esparcimiento. Sin embargo entre semana también hay algunos momentos sociales, en especial en los hogares donde hay miembros que pasan gran parte del día en casa: la visita de un amigo, el compartir un cafecito bordando con una amiga, la visita de una vecina, son interacciones que se dan en el espacio de la sala comedor.

La última interacción identificada en este espacio es la individual: esta se lleva a cabo por los miembros de la familia que permanecen mayor tiempo en el hogar, bien sea porque son amas de casa o porque trabajan allí; el comedor se convierte en un espacio para desarrollar otro tipo de actividades como trabajar en el computador, hacer cuentas entre otros. Por su parte la sala, en especial el sofá, es un lugar que permite un espacio para sí mismo: de relajación, de tranquilidad y confort para los que permanecen gran parte del día en el hogar, pero también para los que llegan del trabajo o incluso los que se encuentran solos en el espacio en algún momento. "Cuando estoy trabajando y me quiero relejar, liberar la mente me siento en el sillón y me tomo un cafecito. O si estoy solo a veces, me sirvo un café y me disfruto ese momento de ocio, y en ese sillón que es cómodo me permite relajarme" (Hog. 6).

Esta interacción individual da cuenta de esos momentos que comienzan a hablar de una vida privada e íntima que se entrelaza con una vida en común de la familia, la pareja y los amigos, en donde los espacios comienzan a delimitar esa línea delgada que hay entre ellas. En el caso de los hogares 
estudiados uno de los espacios favoritos del hogar es la habitación dado que allí tienen sus pertenencias y pueden descansar, relajarse, ver televisión, especialmente cuando llegan del trabajo y luego de haber compartido un rato con los familiares; es un espacio propio. Sin embargo cuando los miembros de la familia permanecen gran parte del día en el hogar, su espacio favorito es la sala comedor; allí realizan diferentes actividades como trabajar, estudiar (comedor) relajarse y compartir con otras personas e incluso con las mascotas (sala).

La apropiación del espacio depende del tipo de interacción que se vaya a tener, si se quiere disfrutar de un momento más privado o si se desea compartir con familiares o amigos: "es que es dependiendo del día, por ejemplo, si quiero compartir con la gente me gusta mucho hacernos acá en el mueble, pero si ya estoy muy cansada y eso, ya como que quiero estar sola, entonces me voy para mi alcoba" (Hog. 3). Y esos procesos dinámicos de interacción van cargando de significado los espacios por las experiencias vividas, las emociones y sentimientos generados.

Entendido de esta forma, el entorno "apropiado" deviene y desarrolla un papel fundamental en los procesos cognitivos (conocimiento, categorización, orientación, etc.), afectivos (atracción del lugar, autoestima, etc.), de identidad y relacionales (implicación y corresponsabilización). Es decir, el entorno explica dimensiones del comportamiento más allá de lo que es meramente funcional. (Vidal y Pol, 2005, p. 284)

\section{Los objetos}

En los hogares estudiados se pudo apreciar que las características para valorar los objetos van desde las funcionales que permiten resolver problemas de espacio, tiempo y adaptabilidad pasando también por aspectos más estéticos, emocionales, de experiencias y el disfrute de estos. Si bien es cierto que las características funcionales de los objetos aportan a la significación de los 
espacios, también son las nuevas dinámicas de apropiación las que comienzan a ser relevantes: "el objetivo es tener un espacio de bienestar sensitivo, de placeres estéticos y sensoriales, de convivencia y personalidad" (Lipovetsky, 2013, p. 287).

En el ámbito funcional se encontró que los objetos son valorados por su tamaño; el cual les permite adaptarse a los espacios actuales por su practicidad para ordenar los espacios, por las funciones que cumple (sentarse, acostarse, estudiar, relajarse trabajar entre otros) y por la facilidad de poder limpiarlos. "Es práctica porque proporciona la facilidad de sentarse, compartir, fácilmente se puede mover, cambiar, limpiar, por eso la veo práctica" (Hog. 1). Aquí se encuentran el sofá, las mesas, el bife y otro tipo de muebles que permiten ordenar los espacios y guardar objetos; tal como refiere Baudrillard (1978): "ya no se trata de implantar un teatro de objetos, o de crear una atmósfera, sino de resolver un problema, de proporcionar la respuesta más sutil a un traslapamiento de datos, de movilizar un espacio" (p. 25).

Sin embargo la decoración y los objetos son muy importantes porque le dan una identidad al grupo familiar y porque de cierta manera reflejan sus gustos, preferencias e incluso personalidad. En los hogares donde la mamá es ama de casa, la decoración, los objetos y muebles de la sala comedor están influenciados por los gustos de ella; a diferencia de los hogares donde la mamá trabaja o donde son grupos de amigas o hermanas, en los que se puede apreciar que los gustos son compartidos. En el ámbito estético se evidencia la inclinación por los gustos de lo moderno y minimalista, así como los colores y materiales de los objetos combinen con los espacios y muebles. Los gustos personales se pueden apreciar en la sala comedor, su configuración y objetos.

Los objetos que permiten interacción, placeres individuales, experiencias familiares y sociales son altamente valorados por los miembros del hogar; el 
poderse sentar, descansar, compartir con otros, genera sentimientos agradables y da una significación especial a estos. Algunos objetos cobran mayor valor cuando llegan las visitas; como el sofá que permite sentarse cómodamente, que a veces está ubicado de tal forma que se pueda tener la mejor vista hacia la ventana, que permite bordar plácidamente y disfrutar de un tinto, que está cerca de la cocina para no perder la interacción mientras se prepara algo para compartir o que en algunas ocasiones está frente al televisor y permite que varios invitados se acomoden para disfrutar de una buena película o de un plan de karaoke y vídeos.

En el ámbito sentimental encontramos los objetos de decoración que poseen un gran valor porque fueron un regalo de un familiar o amigo o porque fue diseñado por algún miembro de la familia o son objetos y recuerdos de viajes realizados. También están aquí los objetos que propician experiencias individuales o grupales placenteras como relajarse, tener un espacio confortable, compartir con los amigos. Con respecto a lo espiritual se observó que los objetos relacionados son relevantes en la configuración del espacio de la sala comedor; se encontraron cuadros y figuras religiosas y objetos de otras culturas con un valor simbólico de espiritualidad, energía, armonía, ambiente, vibraciones; todo esto, de acuerdo a las creencias de los hogares.

Por último, se encontró que los objetos cobran valor por el bienestar sensitivo que proporcionan: armonía, amplitud, vida, calidez. "Yo siempre pienso que los muebles le da mucha vida a los espacios, la casa siempre ha sido acogedora, pero ahora con los muebles, se tornó más cálida, más amañadora" (Hog. 2). Tal es el caso del color y tamaño de los muebles, los espejos y cuadros grandes para generar mayor percepción de amplitud, las flores y plantas que le dan vida a los espacios, los muebles y barras que permiten optimizar el espacio. De igual manera la disposición dinámica de los objetos aporta nuevas percepciones, ver los objetos de manera diferente con cierta periodicidad 
permite renovación a los espacios: "a mí me gusta mucho renovar, en todas las cosas, por ejemplo, en este comedor, yo sé que es el mismo, pero si lo movemos y le cambiamos la ubicación, como que da un nuevo aire, nuevas energías y todo no sé" (Hog. 3). El hecho de comprar nuevos muebles y objetos da también esa percepción de un nuevo ambiente, moderno y estético; de hecho, las principales aspiraciones de compra de nuevos objetos se basan en la decoración: alfombras de moda, cuadros, mesas que hagan juego con los muebles y con el color de la decoración.

Los objetos más usados son a la vez los considerados como indispensables dado que permiten un placer sensorial: las sillas y mesa del comedor para disfrutar cómodamente de las comidas y compartir con familiares e invitados, además de trabajar y estudiar; los muebles y especialmente el sofá que permiten sentarse cómodamente, tomar una siesta, atender a la visita, relajarse, tener un momento de esparcimiento; también los muebles que permiten guardar y exhibir objetos que ayudan a optimizar y ordenar el espacio para dar una sensación de bienestar y armonía.

\section{Prácticas}

En las prácticas para vestir la sala comedor se encontró que han ido evolucionando a medida que los espacios han cambiado y que las configuraciones de los hogares también lo han hecho.

Al mismo tiempo que cambian las relaciones del individuo con la familia y con la sociedad, cambia el estilo de los objetos mobiliarios. Sofás cama, camas de rincón, mesas bajas, estanterías, son elementos que sustituyen al antiguo repertorio de muebles. La organización cambia también: la cama se convierte en sofá cama, el aparador y los roperos en alacenas ocultables. Las cosas se repliegan y se despliegan, desaparecen, entran en escena en el momento deseado. No cabe duda que estas innovaciones no constituyen de ninguna manera una improvisación libre: las más de las veces, esta mayor movilidad, conmutabilidad y oportunidad no es sino el resultado de una adaptación forzosa a la falta de espacio. (Baudrillard, 1978, p. 15) 
Los muebles y objetos son pensados en el espacio disponible, los colores, formas y estilos tratan de conservar una estética moderna muy acorde con los gustos personales de los miembros de la familia y sobre todo de quienes toman la decisión de compra. Los objetos antiguos y tradicionales van siendo relegados o reemplazados, la restauración no es una práctica común: "si yo traslado la estética de cómo estaba decorada mi casa, a este apartamento, me va a quedar nada que ver con el espacio, vamos a quedar muy llenas muy saturadas. También consideramos que las tendencias y los gustos han cambiado" (Hog. 4).

Nuevas prácticas también han sido instauradas con relación a la nueva configuración de los espacios: la sala comedor como un espacio abierto y compartido; la cocina muy cerca e incluso integrada a la sala comedor; la sala que no necesariamente tiene mesa de centro; esto ha llevado también a nuevos usos de los objetos que en sus casas de niños no eran comunes como, por ejemplo, estudiar o trabajar en el comedor, tomar una siesta en el sofá, que la cocina este visible para la visita y tan cerca a la sala que se puede atender desde allí: "que yo me acuerdo también mucho esos espacios eran exclusivos, o sea no se acuesta a dormir en el sofá, y no se ponga hacer tareas en el comedor, el comedor es para comer, la sala es para la visita, nada más" (Hog. 3). Hoy se han extendido las funciones de la sala comedor y se permiten diversas interacciones familiares, individuales y sociales.

Se pudieron apreciar algunos comportamientos heredados sobre todo en las amas de casa tales como el tener manteles bordados a mano, la disposición en la mesa y la ubicación del papá en la cabecera o la forma de servir las comidas. En los miembros de la familia más jóvenes estos comportamientos no se aprecian, incluso manifestaron no querer heredar las formas de decorar o de disponer algunos objetos que consideran tradicionales. 
Con respecto a las prácticas de vestir la sala comedor cuando llega la visita se evidenciaron dos aspectos fundamentales: el interés de lucir un espacio ordenado y limpio porque este espacio es la cara visible del hogar; sin embargo al momento de disponer el espacio de manera diferente, guardar objetos o sacar otros, no se evidenció que esta práctica fuera común; de hecho, fue reconocida como una práctica de sus mamás o abuelos pero que ya no permanece. Aquí prima más el sentirse a gusto con el espacio, los objetos y la estética de la sala comedor, además de la practicidad de los objetos y su funcionalidad en todos los momentos. "Cuando yo sé que va a venir alguien me gusta que todo esté limpio, pero no guardo nada, todo permanece igual, si vienen muchas personas guardo alguna mesa por mejorar el espacio" (Hog. 6). Como refiere Lipovetsky (2013):

para una cantidad reciente de personas no se trata ya de "causar impresión" o de "darse aires" sino de poner estilo en la propia casa y darle carácter. Lo importante no es proclamar que se tiene una posición superior, sino darse placer, rodearse de un entorno creativo e imaginativo, dar un aspecto singular a la casa donde uno quiere sentirse bien. (p. 287-288)

Cuando se trata de decidir sobre cómo vestir la sala comedor, en su gran mayoría, intervienen varios miembros del hogar; los gustos son compartidos, se escuchan las opiniones de todos para comprar y distribuir los objetos y muebles en el espacio. Únicamente se ve la diferencia en los hogares que tienen ama de casa, en donde esta es la que mayor influencia tiene en las decisiones de compra. En cuanto a las ocasiones de compra de objetos o mobiliario se dan a partir de una mudanza de vivienda en el que cambiar los muebles y objetos aportan a crear una vida nueva; o con un cambio de cortina para que armonice con la decoración y colores del espacio; también se da con el cambio de muebles y objetos para renovar, para tener un estilo más moderno. Cambiar con frecuencia la disposición de los objetos es una práctica muy común en los hogares estudiados: a veces se hace teniendo en cuenta su criterio propio o en ciertas ocasiones teniendo como referentes salas 
comedores de conocidos. El renovar no necesariamente implica tener nuevos muebles y objetos sino distribuirlos de manera diferente en el espacio, "si el papel del mobiliario actual prevalece hoy sobre el del "estilo" es sobre todo por el gusto creciente por los ambientes creativos, las novedades y el placer que se obtiene renovando el entorno en función de sus propios deseos" (Lipovetsky, 2013, p. 288).

Con respecto a prácticas inapropiadas en los hogares estudiados son aceptables otro tipo de comportamientos que implícitamente eran inadecuados en una sala comedor, por ejemplo: tomar la siesta en el sofá; tener un televisor; estudiar en el comedor entre otros; lo inapropiado ahora tiene que ver más con el gusto. "Aunque es un tema de gustos, hay gente que le gusta el equipo grande, a mí eso me parece inapropiado, me gustaría algo más pequeño, pero es cuestión de gustos más que inapropiado, por el tamaño. Si yo tuviera solo sala le podría dar cabida al equipo porque seguro tendría más espacio" (Hog. 5).

Finalmente una práctica muy común en los hogares estudiados es el compartir el espacio de la sala con las mascotas, incluso tener muebles y objetos de estas de manera permanente en este espacio y que una de las interacciones relevantes sea precisamente con ellas. "Mi espacio favorito es la sala, en este sillón en que estamos sentadas, me siento porque es muy rico, la vista me gusta, tengo una gata que se llama Carola entonces nos sentamos las dos aquí, a leer, o a oír un audio, o a mirar algo en el computador" (Hog. 1). Un criterio fundamental para configurar los espacios es precisamente la mascota, su cuidado y comodidad, incluso en la decisión de compra de nuevos objetos y muebles esta cobra gran relevancia. Y así en la medida en que cambian las relaciones familiares, cambia el mobiliario de la sala comedor; las relaciones de los individuos con las mascotas también permean las nuevas prácticas de consumo y apropiación de los espacios. 


\section{Conclusiones}

La investigación evidencia que no solo el diseño actual de los espacios y las nuevas dinámicas familiares dan cuenta de los cambios en la apropiación de la sala comedor moderna, sino también los significados y los vínculos con los objetos. Se evidencia como el consumo siendo un conjunto de procesos socioculturales, de apropiación y uso de los objetos, es además un asunto de transferencia de significados en constante transformación y resignificación; el cual es inherente a los objetos, pero especialmente a las personas que hacen esa transferencia. En este sentido el consumo de los espacios modernos, y en especial de la sala comedor, no ha sido ajeno a tales transformaciones; hoy por hoy, este ha pasado de una relación funcional con los objetos a una relación de placer en la que se traslapan el disfrute individual y el colectivo. De igual manera denota una relación más íntima donde estas prácticas de consumo cada vez más individualizadas han generado cambios en las dinámicas familiares, la apropiación personalizada del espacio y el significado de los objetos.

En la configuración de la sala comedor moderna se presta mayor atención a la decoración, al ambiente, a la personalización como elementos de confort visual, de bienestar sensitivo y estético que aportan a los placeres sensoriales. Si bien es cierto que los objetos han sido valorados por su aporte a la practicidad, ahorro de espacio y de tiempo, hoy las cualidades de estos también son estéticas, hedonistas y sensitivas. La decoración de este espacio se ha alejado del imperativo de mostrar una posición en la sociedad para moverse hacia una mezcla de objetos, estilos y ambientes creativos en los que el gusto por lo nuevo y moderno y el placer de cambiar son una constante. Las normas colectivas ya no son un impedimento para el desarrollo de nuevas prácticas de vestir la sala comedor, que dan cuenta de una proyección de identidad donde se da la puesta en escena del individuo y la familia. Los objetos que visten la sala comedor también materializan el confort, representado por 
muebles fáciles de mover, ligeros y ahorradores de espacio, privilegiando multifuncionalidad y adaptabilidad a la sala comedor moderna. Esta a veces se convierte en habitación, en el lugar de convivencia y en el centro del hogar que permite intimidad, plenitud personal y coexistencia.

La apropiación de la sala comedor es una dinámica constante donde los individuos hacen 'suyo' este espacio en la medida que lo transforman y modifican no solo a través del uso y disposición de los objetos sino de establecer una relación con estos, de generar unas vivencias que dejen improntas y apego al espacio. Modificar el espacio permite, en últimas, cargarlo de significación y de símbolos como indicadores de identidad. Las prácticas para vestir la sala comedor son esas acciones que transforman el espacio a través de los objetos que se cargan de significado haciendo del individuo el actor y trasformador del espacio que proyecta su imagen, pero que además busca ese bienestar estético y sensitivo; ese placer y disfrute colectivo o individual que se ha venido resignificando de la misma forma que los espacios y las configuraciones de la familia y la sociedad.

\section{Referencias}

Bal, M. (2009). Conceptos viajeros en las humanidades: una guía de viaje. Murcia, España: CENDEAC.

Baudrillard, J. (1978). El sistema de los objetos. Ciudad de México, México: Siglo XXI Editores.

Cano, O.A. (2012). Mercancía y hogar: la casa como no-lugar. AIBR. Revista de Antropología Iberoamericana, 7 (1), 99-119.

Chávez, J.D. (2010). El espacio doméstico tras el soporte arquitectónico: claves para comprender el sentido multidimensional de lo íntimo en el dominio del hogar. Dearq, 7, 6-17. 
Douglas, M. (1979). El mundo de los bienes. Ciudad de México, México: Grijalbo.

Guber, R. (2001). La etnografía: método, campo y reflexividad. Bogotá, Colombia: Grupo Editorial Norma.

Lévi-Strauss, C. (1988). Las estructuras elementales del parentesco. Barcelona, España: Paidós.

Lipovetsky, G. (2006). La felicidad paradójica. Barcelona, España: Anagrama.

Lipovetsky, G. (2013). La estatización del mundo. Barcelona, España: Anagrama.

Medina, F. (2005). La dimensión comunicativa del objeto: una propuesta de análisis. Iconofacto, 1 (89), 109-115.

Pineda, R.A. (2016). La sala del hogar como contexto objetal: planteamiento de una categoría para el estudio de la cultura material. Iconofacto, 12 (18), 70-95.

Sassatelli, R. (2012). Consumo, cultura y sociedad. Buenos Aires, Argentina: Amorrortu.

Vidal, T.M. y Pol, E.U. (2005). La apropiación del espacio: una propuesta teórica para comprender la vinculación entre las personas y los lugares. Anuario de Psicología, 36 (3), 281-297.

Como citar: Montoya, V. y Torres, I.C. (2019). Valor simbólico de los objetos para vestir la sala comedor del hogar y su aporte a la apropiación de los espacios. Revista KEPES, 16 (19), 295-315. DOI: 10.17151/ kepes.2019.16.19.12 